\title{
Erratum zu: CSR und Energiewirtschaft
}

Alexandra Hildebrandt und Werner Landhäußer

Erratum zu:

\section{A. Hildebrandt und W. Landhäußer (Hrsg.), CSR und Energiewirtschaft, Management-Reihe Corporate Social Responsibility, https://doi.org/10.1007/978-3-662-59653-1}

Die Originalversion der Titelei wurde korrigiert: Auf Seite X wurde das Copyright des Fotos korrigiert.

Kapitel 2: In der Originalversion wurden versehentlich folgende Autorkorrekturen nicht korrekt ausgeführt.

Auf Seite 15 in der 5. Textzeile musste folgendes gelöscht werden: mehr im.

Auf Seite 16 in der 13. Textzeile musste folgendes „Darauf der“ zu „Darauf baut der“ geändert werden.

Auf Seite 19 musste die Autorenbiographie korrigiert werden und lautet nun:

Dr. Colin von Ettingshausen, geboren 1971 in Düsseldorf. Seit 2012 kaufm. Geschäftsführer und Arbeitsdirektor der BASF Schwarzheide GmbH. Mitglied der Tarifkommission Bundesarbeitgeberverband Chemie, stellv. Vorsitzender des Verwaltungsausschuss Agentur für Arbeit Cottbus sowie Mitglied der Vollversammlung IHK Cottbus. Studium der Betriebswirtschaftslehre in Dortmund und Plymouth (UK). Studium der International Relations und Economics in Oxford. Von 1999 bis 2012 ver-

Die aktualisierte Version des Buches finden Sie unter https://doi.org/10.1007/978-3-662-59653-1

https://doi.org/10.1007/978-3-662-59653-1_2 
schiedene Positionen in Vertrieb und Marketing für Autoreparaturlacke des BASF Unternehmensbereichs Coatings Solutions in Münster, Johannesburg, Salzburg und Yokohama. Silbermedaille im Zweier ohne Steuermann bei den Olympischen Spielen von Barcelona 1992. Ruder-Weltmeister im Deutschlandachter in Prag 1993. Teilnehmer im Zweier ohne Steuermann bei den Olympischen Spielen von Atlanta 1996. 